\title{
Sistemas de Nanopartículas Poliméricas I: de Biodetección y Monitoreo de Glucosa en Diabetes a Bioimagen, Nano-Oncología, Terapia Génica, Ingeniería de Tejidos / Regeneración a Nano-Odontología
}

\author{
Polymer Nanoparticle Systems: from Biodetection and Glucose Monitoring in Diabetes to \\ Bioimaging, Nano-Oncology, Gene Therapy, Tissue Engineering/Regeneration to Nano-Dentistry
}

\author{
Madelein C. Urrejola ${ }^{1,2}$; Liliam V. Soto ${ }^{1}$; Consuelo C. Zumarán ${ }^{1,3}$; Juan Pablo Peñaloza ${ }^{1,3}$; \\ Beatriz Álvarez ${ }^{1,3}$; Ignacio Fuentevilla ${ }^{1,3}$ \& Ziyad S. Haidar ${ }^{1,3,4,5,6}$
}

URREJOLA, M. C.; SOTO, L. V.; ZUMARÁN, C. C.; PEÑALOZA, J. P.; ÁLVAREZ, B.; FUENTEVILLA, I. \& HAIDAR, Z.S. Sistemas de nanopartículas poliméricas: de biodetección y monitoreo de glucosa en diabetes a bioimagen, nano-oncología, terapia génica, ingeniería de tejidos / regeneración a nano-odontología. Int. J. Morphol., 36(4):1490-1499, 2018.

RESUMEN: Nanotecnología es la ciencia que involucra la síntesis de materiales en escala entre 1-100 nm (nanomateriales) es aplicable en diferentes áreas tales como medio ambiente, electrónica, alimentos, energía, entre otros. Los campos que serán relevantes dentro de esta revisión y explicados en detalle son la nanomedicina y la nano-odontología. Actualmente, en estas áreas los tres principales temas en desarrollo son específicamente en el sub-área de la nanobiotecnología y corresponden a: sensorización (biosensores/biodetección), diagnóstico (biomarcadores/bioimagen) y transportes de genes, proteínas o fármacos (sistemas de intercambio controlado en blancos sistémicos versus localizados). También se han presentado avances en bioaplicaciones como modelamientos de membranas, marcaje celular, entrega de agentes a blancos específicos, estrategias para prevención de enfermedades, ingeniería de tejidos, regeneración de órganos, estrategias de inmunoensayos y nano-oncología. Este artículo de revisión pretende abordar algunos de los aportes más relevantes, que tienen algunos de los trabajos recientes, sobre los sistemas de nanopartículas, principalmente aquellos dirigidos a terapias en áreas como diabetes, nano-oncología, terapia de fármacos y genes, mediante la técnica layer-by-layer y autoensamblado, muy utilizados también en ingeniería de tejidos y regeneración tisular, junto a un breve resumen de los avances que existen en el campo de la nano-odontología.

PALABRAS CLAVES: Glucosa; Quantum dots; Polímeros; Regeneración; Nanotecnología.

\section{INTRODUCCIÓN}

Durante los últimos años, la nanotecnología se ha utilizado en diversos ámbitos de la biomedicina; por ejemplo, en el caso de enfermedades como diabetes, poseen un potencial valioso para mejorar el cuidado de los pacientes. La nanotecnología se ha utilizado en enfoques no invasivos de la administración de fármacos como insulina, y en un futuro próximo, la síntesis de vacunas más eficaces que sirvan como terapia génica para tratar, entre otras, esta enfermedad.
Además, las nanopartículas se están desarrollando como agentes de contraste de imágenes para ayudar en el diagnóstico precoz de la diabetes tipo 1. Junto con esto, se están incorporando nanosensores de glucosa en dispositivos implantables que permiten un seguimiento más preciso y en tiempo real, de los niveles de glucosa en sangre, proporcionando la base para nanopartículas que respondan a diferentes niveles de glucosa e imiten mejor las necesidades fisiológicas de insulina (Veiseh et al., 2015).

\footnotetext{
${ }^{1}$ BioMAT'X, Universidad de los Andes, Las Condes, Santiago, Chile.

${ }^{2}$ Facultad de Odontología, Universidad Andrés Bello, Santiago, Chile.

${ }^{3}$ Plan de Mejoramiento Institucional (PMI) en Innovación I+D+i, Universidad de Los Andes, Las Condes, Santiago, Chile.

${ }^{4}$ Programa de Doctorado en BioMedicina, Facultad de Medicina, Universidad de Los Andes, Las Condes, Santiago, Chile.

${ }^{5}$ Centro de Investigación e Innovación Biomédica, Universidad de Los Andes, Las Condes, Santiago, Chile.

${ }^{6}$ Facultad de Odontología, Universidad de los Andes, Las Condes, Santiago, Chile.
} 
URREJOLA, M. C.; SOTO, L. V.; ZUMARÁN, C. C.; PEÑALOZA, J. P.; ÁLVAREZ, B.; FUENTEVILLA, I. \& HAIDAR, Z. S. Sistemas de nanopartículas poliméricas I: de biodetección y monitoreo de glucosa en diabetes a bioimagen, nano-oncología, terapia génica, ingeniería de tejidos / regeneración a nano-odontología. Int. J. Morphol., 36(4):1490-1499, 2018.

Las vacunas son importantes herramientas en el tratamiento y prevención de enfermedades, tienen como objetivo prevenir o tratar enfermedades a través de la manipulación de la respuesta inmune, promoviendo la inmunidad o la tolerancia (Irvine et al., 2013). Su eficacia se ve afectada por causas tan diversas como el tamaño de la partícula de la vacuna, la vía de administración e incluso las propiedades químicas de superficie, variables que pueden incidir en la cinética de exposición mediante el control del tráfico fisiológico a diversos tejidos y células, así como a compartimentos intracelulares. Si bien, existe una creciente apreciación de que estas variables afectan significativamente la calidad y la magnitud de la respuesta inmune, sigue habiendo poca comprensión de los mecanismos subyacentes en juego (Irvine et al.).

Por otra parte, el cáncer es una de las enfermedades que presenta mayores complicaciones, incluso se le considera como una enfermedad autónoma de las células, cuya complejidad puede ofrecer un gran obstáculo que dificulta el progreso en la salud de los pacientes. Aunque se han hecho grandes avances en la comprensión de la etiología del cáncer, la capacidad de entregar resultados duraderos a los pacientes sigue siendo limitada; por lo tanto, hay un interés sustancial en la creación de formas más efectivas para aprovechar la inherente actividad antitumoral de las células del sistema inmune para tratar tumores resecados de forma parcial o aquellos inoperables (Stephan et al., 2015). Es aquí donde la nanotecnología aparece como una herramienta que puede mejorar la eficacia de moléculas inmunomoduladoras, alterando su co-localización, biodistribución y liberación cinética (Goldberg, 2015).

Los liposomas y las partículas compuestas de polímeros biodegradables como el PLGA se han estudiado durante muchos años como portadores de vacunas, pero sufren de múltiples limitaciones, que incluyen los bajos niveles de antígeno atrapado, la necesidad de disolventes orgánicos en el procesamiento, y la desnaturalización de las estructuras tridimensionales de los antígenos, que son críticas para generar las respuestas inmunes humorales (Irvine et $a l$.). Por otra parte, en el campo de la ingeniería tisular, las nanopartículas han sido utilizadas en el tratamiento de quemaduras, proporcionando factores de crecimiento a la zona afectada y andamios de ingeniería tisular, considerando la aplicación de células madre de manera exógena para ayudar a la regeneración de la piel (Mofazzal Jahromi et al., 2017).

\section{Aplicaciones biomédicas de nanoestructuras}

Bio-detección en Diabetes y monitoreo del nivel de Glucosa. Según la Asociación Americana de Diabetes, diabetes mellitus se define como una enfermedad crónica caracteri- zada por hiperglucemia causada por un defecto en la acción y/o secreción de la insulina, esta enfermedad posee complicaciones a largo plazo tales como nefropatía, neuropatía, retinopatía y cataratas. La nanotecnología ha facilitado el desarrollo en la investigación sobre la diabetes, permitiendo la creación de nuevos sistemas de medición y nuevas modalidades para administrar insulina que tienen por objetivo mejorar drásticamente la calidad de vida de los diabéticos. Recientemente, se han realizado avances en el campo de los biosensores de glucosa, cuyos componentes a nanoescala aumentan la sensibilidad, la respuesta temporal y la monitorización in vivo continua de los niveles de glucosa en la sangre.

Existen tres clases principales de moléculas de detección de glucosa que se están utilizando para diseñar sensores de glucosa basados en nanopartículas, éstos incluyen glucosa oxidasa, proteínas de unión a la glucosa y moléculas pequeñas de unión a la glucosa. Cuando se combinan con nanopartículas diseñadas como transductores, estas moléculas de detección específicas de glucosa permiten el diseño de nuevos tipos de sensores que tienen el potencial de ser más amigables con el paciente, proporcionar mediciones rápidas y mejorar la precisión (Veiseh et al.).

Actualmente, existen otros sensores de glucosa con nanopartículas de metales y carbono, que aumentan la sensibilidad de la medición y liberan insulina a "circuito cerrado" en respuesta a los niveles fluctuantes de glucosa en la sangre (BGLs), mejorando la salud y calidad de vida de los pacientes que presenten éstas patologías (DiSanto et al., 2015). Muchas de estas estrategias han demostrado una liberación pulsátil de insulina in vitro en respuesta a cambios en las concentraciones de glucosa y de glicemia en roedores de 10 a 295 días después de la administración de una dosis única (Veiseh et al.). Sin embargo, los sistemas basados en la glucosa-oxidasa pueden tener velocidades de respuesta lentas, y a veces impredecibles a las variaciones de las concentraciones de glucosa, debido a cambios en la temperatura o concentración de oxígeno que posteriormente afectan a la traducción de los cambios en la concentración de glucosa (Veiseh et al.).

A medida que avanza la diabetes, hay una reducción en la masa de las células b y con ello de la producción y secreción de insulina, una cuantificación de las células b que aún se encuentran funcionales podría ser útil para mejorar las terapias y dirigirlas hacia este tipo celular. Aunque se han hecho avances importantes en el desarrollo de sondas que forman imágenes para monitorizar la inflamación y la biomasa de células b, sigue siendo una necesidad desarrollar sondas molecularmente dirigidas, que puedan 
URREJOLA, M. C.; SOTO, L. V.; ZUMARÁN, C. C.; PEÑALOZA, J. P.; ÁLVAREZ, B.; FUENTEVILLA, I. \& HAIDAR, Z. S. Sistemas de nanopartículas poliméricas I: de biodetección y monitoreo de glucosa en diabetes a bioimagen, nano-oncología, terapia génica, ingeniería de tejidos / regeneración a nano-odontología. Int. J. Morphol., 36(4):1490-1499, 2018.

informar directamente sobre la funcionalidad de islotes in vivo. El desarrollo de biomarcadores únicos que sean específicos para la superficie de las células b, y que puedan controlar el estrés o la disfunción de éstas, podría ayudar a acelerar la evaluación clínica de terapias para promover la salud y la supervivencia de las células b, así como la estratificación de los pacientes según el estado de la enfermedad para la aplicación de terapias dirigidas (Veiseh et al.).

El desarrollo y la prueba de nanosensores de glucosa se encuentra en una etapa temprana para la evaluación in vivo, hasta el momento, sólo se han llevado a cabo estudios en animales. Se han desarrollado tecnologías no enzimáticas para los nanosensores, que pueden traducirse fácilmente y convertirse en sensores continuos de supervisión de glucosa de próxima generación, con menos variabilidad que los sensores actuales basados en la glucosa oxidasa. Los nanosensores no invasivos con lecturas ópticas o fluorescentes son los más prometedores para reemplazar el estándar actual de detección manual de glucosa, basado en la punción de los dedos; tales sensores basados en lecturas ópticas han generado un interés considerable porque no requieren de una batería o un catalizador enzimático y pueden ser diseñados para proporcionar lecturas ultrarrápidas a velocidades que son significativamente más altas que los sensores actuales basados en electrodos. En general, los dispositivos de detección se construyen uniendo tres componentes clave: un detector que mide las concentraciones de glucosa en sangre; un transductor que convierte mediciones en señales de salida y un reportero, que procesa la señal generada en datos que pueden entonces ser interpretados por el paciente o el médico.

Además, se han utilizado células madre mesenquimales (CSM) para el tratamiento de la diabetes mellitus tipo 1. Sin embargo, la distribución in vivo y los efectos terapéuticos de CSM trasplantadas no están completamente definidos. Se ha reportado la síntesis de QuantumDots de CdSe/ZnS como marcadores, con el objetivo de ser usados para formar imágenes de fluorescencia, que tienen como fin una terapia sobre los tejidos del páncreas. En modelos de ratas con diabetes tipo 1 se evaluó la biocompatibilidad y concentración apropiada de QuantumDots (QDs) para marcar CSMs (Liu et al., 2015), luego se observaron utilizando el sistema Bruker In-Vivo F PRO y se monitorearon los niveles de glucosa en sangre durante 8 semanas. Los resultados mostraron que los puntos cuánticos $\mathrm{CdSe} / \mathrm{ZnS}$ preparados poseían una buena biocompatibilidad. Por lo tanto, las MSC marcadas con $\mathrm{CdSe} / \mathrm{ZnS}$ pueden dirigirse a tejidos de páncreas in vivo, y reducir significativamente los niveles de glucosa en sangre, teniendo una aplicación potencial en terapia de pacientes diabéticos en un futuro próximo.
Por otra parte, Ancla et al., (2011) sintetizaron microgeles estructurados tipo multicapa, que poseen la propiedad de tener una respuesta múltiple para encapsular insulina, que tienen una liberación controlada proporcional a las concentraciones de glucosa (Ancla et al.,). Otro estudio realizado por Mukhopadhyay et al. (2015) logró la encapsulación de insulina en nanoesferas de alginato/ quitosano, que presentaban biocompatibilidad y ausencia de citotoxicidad, logrando con éxito el encapsulamiento del $85 \%$, pudiendo ver in vivo su efecto hipoglicemiante (Mukhopadhyay et al.). Se han propuesto también sensores amperométricos no enzimáticos basado en electrodo modificado de nanoparticulas de $\mathrm{CuO}$, grafeno y nanofibras de carbono, lo que conduce a mayores áreas de superficie activa y transferencia de electrones más rápida para el sensor de glucosa, el cual también tiene aplicaciones para la medición de la glucosa salival, cuya respuesta es rápida, altamente sensible y estable en el tiempo, esto lo convierte en una ruta no invasiva para medir los niveles de glucosa tanto en pacientes sanos como diabéticos (Ye et al., 2013).

Bioimagen: Membrana celular / Etiquetado Intracelular. El término bioimagen considera la observación in vivo de estructuras como proteínas y ácidos nucleicos, a partir de sondas luminiscentes, como nanocristales semiconductores u otros tipos de nanopartículas.

Las nanoestructuras poseen variadas propiedades entre las que se encuentran su simple funcionalización y conjugación con biomoléculas, que han contribuido tanto en el diagnóstico precoz de diversas patologías como: diabetes, cáncer, enfermedad de Alzheimer y accidentes cerebrovasculares, como en el tratamiento y prevención de las mismas (Chatterjee et al., 2014). Investigadores realizaron estudios donde utilizaron nanopartículas compuestas por Au-Cu9S5, Ag2S, además de nanopartículas de conversión ascendente (UCNPs) las cuales son utilizadas como biosensores para bioimagen, a través de la generación de una señal lumínica en dos rangos, dentro del espectro de la luz visible y otro dentro del espectro del infrarrojo, cuyo objetivo principal es utilizarlas en detección de microRNA dual en células vivas, los cuales se encuentran alterados en células cancerosas, permitiendo así evidenciar mutaciones de éste marcador mediante la administración de estas nanopartículas en conjunto a equipos generadores de imágenes, como tomografía computarizada de rayos X (TC), imagen fotoacústica e imagen de luminiscencia NIR-II (Li et al., 2017).

Por otra parte, Chatterjee et al. proponen utilizar la imagen por resonancia nuclear magnética (MIR), ya que posee una alta capacidad de reproducción de órganos. 
URREJOLA, M. C.; SOTO, L. V.; ZUMARÁN, C. C.; PEÑALOZA, J. P.; ÁLVAREZ, B.; FUENTEVILLA, I. \& HAIDAR, Z. S. Sistemas de nanopartículas poliméricas I: de biodetección y monitoreo de glucosa en diabetes a bioimagen, nano-oncología, terapia génica, ingeniería de tejidos / regeneración a nano-odontología. Int. J. Morphol., 36(4):1490-1499, 2018.

Nanopartículas de óxido de gadolinio $\left(\mathrm{Gd}_{2} \mathrm{O}_{3}\right)$, fluoruro de gadolinio $\left(\mathrm{GdF}_{3}\right)$ y fosfato de gadolinio sódico $\left(\mathrm{NaGdF}_{4}\right)$ se han estudiado como agentes de contraste para esta técnica de generación de imágenes, así como también para tomografía computarizada y tomografía por emisión de positrones. Además de nanopartículas cargadas con sustancias con características ópticas, se han utilizado Quantum Dots para detectar sustancias intracelulares, como biosensores proteolíticos y monitorización de liberación de fármacos, donde se puede seguir in vivo las células inyectadas con proteína fluorescente (Field et al., 2017).

Muchos investigadores han utilizado esta nanotecnología para la detección del cáncer y tumores, ejemplo de ello son Jie et al. (2014) quienes sintetizaron un aptámero polimérico con CdSe/ZnS QDs, cuya fluorescencia serviría como una estrategia en ingeniería molecular con la exitosa capacidad de captar imágenes de células tumorales cancerosas in vivo, incluso a largo plazo. Esta estructura tiene mejores capacidades de reconocimiento como diana e internalización celular (Jie et al.).

Nano-oncología. La nano-oncología es la nanotecnología enfocada en desarrollar terapias anti-cancerígenas. Las nanopartículas podrían ser cargadas con compuestos terapéuticos para conseguir una administración local y concentrada de fármacos con un potencial de liberación sostenida cuando se utilizan portadores biodegradables. Su elevada relación superficie-área-volumen permite recubrirlos con diversos ligandos (por ejemplo, anticuerpos o aptámeros) que pueden facilitar la interacción con moléculas afines, incluyendo receptores presentes en la superficie de las células diana.

Usualmente, las células cancerosas se estudian aisladas de su entorno normal, el microambiente tumoral, el cual está compuesto de una gran cantidad de tipos celulares; entre ellos se encuentran las células inmunes, las cuales afectan en gran medida la iniciación, progresión e invasión de la enfermedad, donde la densidad y localización de las células inmunitarias dentro de los tumores son un predictor de la supervivencia del paciente, incluso mejor que los métodos patológicos convencionales (Goldberg). Es por el aporte que tiene este tipo celular en la progresión del cáncer que se ha considerado el despertar las células inmunitarias agotadas como una opción terapéutica. Estudios confirman que estimular la respuesta inmune antitumoral natural de un paciente, puede curar aquellos cánceres refractarios que no han respondido a otros tratamientos (Topalian et al., 2011). Además, los tumores son heterogéneos y están en constante cambio, por lo tanto, las células inmunes pueden generar una respuesta antitumoral coordinada y adaptativa con capacidad de memoria que no es alcanzable utilizando ninguna otra modalidad terapéutica (Goldberg).
Sin embargo, debido a que los tumores suelen evadir el sistema inmune, estos tratamientos han beneficiado solo a una minoría de pacientes. Por ello, se busca lograr una inmunidad antitumoral mejorada, ayudada por nanopartículas capaces de facilitar la acción de células inmunitarias antitumorales gracias al aumento de la captación por parte de las células cancerosas, pero aún no se logra una localización tumoral perfecta por parte de estas, por lo que se busca cambiar el esquema actual (administración de fármacos citotóxicos a células cancerosas) por esta nueva alternativa, que es la administración de fármacos inmunoestimulantes a células inmunitarias antitumorales (Fig. 1). Si bien, es difícil concentrar nanopartículas en un tumor tras una administración sistémica de estas, es posible producir una eficacia tumoral solo con una pequeña carga de células inmune debido a que las células inmunitarias proliferan ampliamente tras su activación.

Estudio de vacunas contra el cáncer. Un campo de investigación en rápido crecimiento es el diseño de materiales sintéticos en vacunas, los cuales se han dirigido principalmente a: (1) órganos, tejidos, células o compartimentos intracelulares diana; (2) co-entrega de señales inmunomoduladoras que controlan la calidad de la respuesta inmune; (3) actuar como reguladores inmunes directos (Irvine et al.). Los avances realizados en el campo de la inmunología celular y molecular apuntan al diseño de nuevas vacunas, que presenten una eficacia mejorada. Irvine $e t$ $a l$. describen los avances recientes en el diseño de materiales sintéticos que son utilizados para dirigir las respuestas inmunes, destacando los éxitos y desafíos en las vacunas profilácticas, terapéuticas e inductoras de tolerancia. Estas vacunas conducen predominantemente a la generación de anticuerpos neutralizantes u opsonizantes, que pueden ser difíciles de lograr en algunas enfermedades.

Existen limitaciones que impiden alcanzar avances importantes en esta área, como las restricciones que presentan los modelos animales y la necesidad de cumplir con las excepcionales normas de seguridad necesarias en materia del desarrollo de vacunas profilácticas diseñadas para ser administradas a poblaciones sanas. Gran parte del esfuerzo actual se centra en el desarrollo de vacunas de subunidades, compuestas por antígenos de proteínas, péptidos o polisacáridos, que se espera cumplan estos estándares de seguridad. Sin embargo, los antígenos purificados son típicamente poco inmunogénicos y deben combinarse con adyuvantes, es decir, materiales que promueven la respuesta inmune, o instruir directamente a las células presentadoras de antígeno (APC) para obtener inmunidad contra el antígeno co-administrado. Es en el diseño de adyuvantes donde la ciencia de los materiales y la ingeniería tienen un segundo papel fundamental y potencialmente crítico en el 


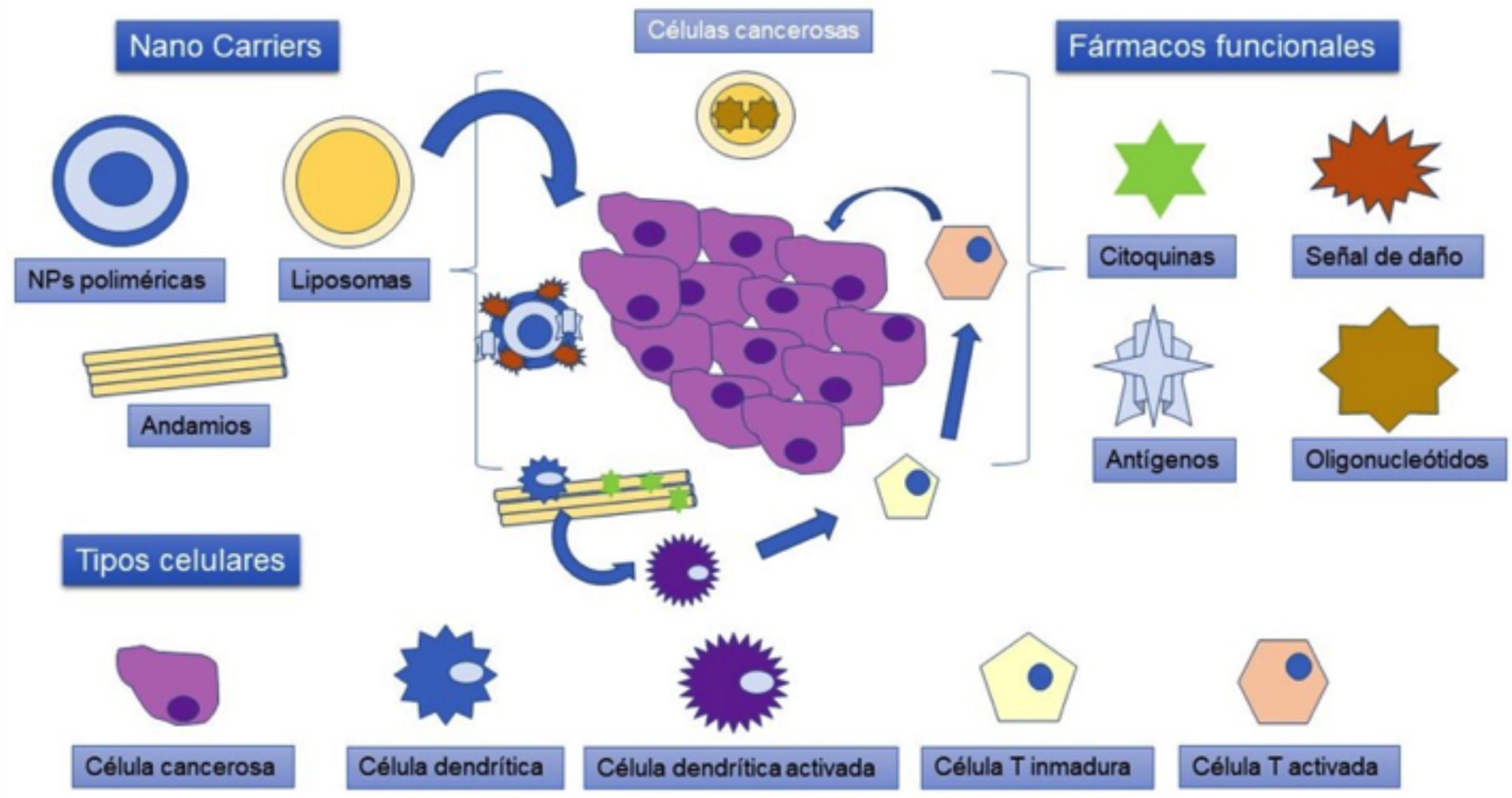

Fig. 1. Aplicaciones de la nanotecnología sobre células inmunitarias. Muestra las aplicaciones de distintos nanosistemas sobre las células del sistema inmune. (1) Administrar antígenos, por ejemplo, a través de NPs poliméricas, (2) Estimular células T directamente a través de células presentadoras de antígenos artificiales, (3) Concentrar compuestos inmunoestimulantes en el microambiente inmunosupresor, a través del uso de andamios y (4) Administrar fármacos de apoyo a las células T en la circulación, por ejemplo, por medio de Liposomas funcionalizados.

futuro de las vacunas, a través del diseño de nuevos materiales inmunoestimulantes y en el desarrollo de sistemas de administración que potencien la respuesta inmune para vacunas de subunidades seguras y eficaces.

Hasta hace muy poco, los únicos adyuvantes aprobados para su uso por la FDA eran las sales de aluminio (denominadas colectivamente alúmen), que se desarrollaron por primera vez en los años veinte. Sin embargo, los avances en la comprensión de las vías moleculares que regulan la detección de microbios por el sistema inmunológico han llevado a establecer una amplia gama de nuevos objetivos para el diseño adyuvante. Por su parte, los biomateriales adaptados ofrecen la posibilidad de activar de forma selectiva los sensores inmunes durante la vacunación, combinados con el control de la cinética de exposición de vacunas en el tejido y los niveles de células individuales. Estos materiales también tienen un enorme potencial como herramientas para lograr una mejor comprensión de las complejidades de la regulación inmune. Por ejemplo, propiedades como el acoplamiento preciso de antígeno o adyuvante; características superficiales que promuevan o prevengan la adsorción de proteínas o acti- vación del complemento; sensibilidad a la degradación endosómica o lisosomal después de la captación celular; y el tamaño - entre otros - todos pueden ser controlados con precisión mediante materiales de ingeniería.

Algunos de estos avances han tenido resultados exitosos, sin embargo, otros no han sido tan promisorios, pero todavía quedan enormes desafíos como consecuencia de nuestra incompleta comprensión acerca de la inmunidad humana (Irvine et al.).

Andamios poliméricos e inmuno-ingeniería. Durante los últimos años se han sintetizado andamios poliméricos e hidrogeles, en los cuales se pueden implantar micro-ambientes modulares adaptados que pueden co-localizar con citoquinas inflamatorias y antígenos antitumorales. Los mecanismos de metástasis ósea relacionada con el cáncer de mama son poco conocidos y ocurren frecuentemente en las etapas avanzadas de la enfermedad. En esta etapa, la enfermedad se considera incurable. Esto se puede atribuir en parte, a la falta de modelos animales apropiados que puedan ser usados para investigar las complejas interacciones célula-hueso en el contexto del cáncer (Quent et al., 2017). 
URREJOLA, M. C.; SOTO, L. V.; ZUMARÁN, C. C.; PEÑALOZA, J. P.; ÁLVAREZ, B.; FUENTEVILLA, I. \& HAIDAR, Z. S. Sistemas de nanopartículas poliméricas I: de biodetección y monitoreo de glucosa en diabetes a bioimagen, nano-oncología, terapia génica, ingeniería de tejidos / regeneración a nano-odontología. Int. J. Morphol., 36(4):1490-1499, 2018.

En un estudio, Quent et al. aplicaron a un modelo de cáncer de mama que presentaba metástasis, dos constructos de tejidos de ingeniería de hueso (TEBCs). Primero compararon un andamio de fosfato de policaprolactona-tricálcico de grado médico, que fue producido mediante modelado de deposición fundida (andamio 1), con un andamio de calcio tubular sintetizado con policaprolactona y cubierta de fosfato, fabricado por electrospinning (andamio 2). Aunque los resultados para el andamio 1 no fueron prometedores, ya que, no fue adecuado para generar una cantidad suficiente de tejido óseo ectópico, debido a una integración ectópica deficiente, el andamio 2, por su parte, mostró una excelente integración en el tejido huésped, lo que condujo a la formación de hueso de manera eficiente (Quent et al.). Por otro lado, para imitar la colonización de células de cáncer de mama al hueso, las células se cultivaron en hidrogeles a base de glicol de polietileno y se implantaron adyacentes a los TEBCs. El análisis histológico indicó que las células de cáncer de mama indujeron una reacción osteoclástica en los TEBCs, demostrando así, similitudes a la metástasis ósea, relacionada con el cáncer de mama observada en pacientes (Quent et al.). Una de las terapias más prometedoras para el tratamiento de muchos cánceres, se basa en el uso de células T, sin embargo, su utilidad clínica en el microambiente tumoral es limitada, debido a la dificultad que presenta la administración de linfocitos dirigidos al sitio del tumor, y la expansión ineficiente de las células en los inmunosupresores (Stephan et al.). La potencia anti-tumoral de los linfocitos trasplantados, se pueden mejorar sustancialmente aplicando bioingeniería, es decir, conteniéndolos en matrices poliméricas diseñadas para suministrar y estimular cuando se colocan en sitios de resección tumoral o cerca de tumores inoperables, particularmente dirigido a tumores avanzados, no resecables o sometidos a resección incompleta (por ejemplo, tumores pancreáticos y cáncer de ovario, tumores cerebrales, sarcomas, mesotelioma y cáncer de mama avanzado).

Stephan et al. describen el uso de un polímero bioactivo, que usado como implante es capaz de suministrar, expandir y dispersar células $\mathrm{T}$ reactivas al tumor. El polímero actúa como un reservorio activo, sobre el cual las células propagadoras se liberan a medida que el material se biodegrada. Por lo tanto, este enfoque puede ser utilizado para tratar tumores inoperables o que han sido removidos de forma incompleta, situando implantes cerca de ellos o en sitios de resección. Se han probado en animales a los cuales se les ha realizado resección de cáncer y se muestran efectivos en las células tumorales, utilizando los lechos de los ganglios linfáticos asociados como vía de acceso al tumor, reduciendo la recidiva, en comparación con los métodos convencionales. Los resultados de Stephan et al. establecen que los andamios biodegradables pueden superar las barreras inmunosupresivas creada por los tumores y dispersar gradualmente el anticuerpo proliferante, en las células T que son albergadas a lo largo del lecho tumoral. Por otra parte, las células $\mathrm{T}$ liberadas por el polímero pueden detener la evolución del cáncer, en un ovario multifocal, mientras que los linfocitos inyectables tumor-reactivos tienen poco efecto curativo (Stephan et al.). Es por esto que la entrega de células $\mathrm{T}$, basadas en andamios pueden ofrecen una opción de tratamiento para aquellos tumores que son inoperables, además de reducir la tasa de recidiva metastásica que se presenta después de las cirugías, por lo tanto, es posible que los andamios con biopolímeros, podrían evocar respuestas inmunitarias antitumorales capaces de eliminar metástasis tumorales generalizadas.

\section{Fármacos, Terapia de Genes, Regeneración Ósea e In-} geniería Tisular. Las nano-formulaciones de medicamentos mejoran las propiedades de los fármacos convencionales y son sitio-específicas, algunas de las más utilizadas para la síntesis de fármacos mejorados son: dendrímeros, nanopartículas poliméricas, liposomas, nano-emulsiones y micelas. Existe una gran cantidad de métodos de síntesis para la preparación de nano-formulaciones que permitan la administración de fármacos en el sistema biológico, la elección del método de síntesis depende del tamaño, la formulación de las partículas, las propiedades bioquímicas del fármaco y el sitio diana. Por ello es importante discutir los métodos de síntesis utilizados para el diseño de las nanoformulaciones y sus aplicaciones en la liberación de los fármacos (Jeevanandam et al., 2016).

Por ejemplo, compuestos como la levofloxacina, una quinolona muy comúnmente utilizada contra bacterias causantes de infecciones del tracto respiratorio, cutáneo y genitounirario, han sido utilizados como nuevos agentes antimicrobianos, que exhiben actividades bactericidas in vitro, con un amplio espectro de acción contra aerobios Gram-positivos y Gram-negativos. El levofloxacino lipofílico (isómero activo de la ofloxacina) ha sido cubierto con capas de Quitosano/Alginato, para la síntesis de nanoesferas. La tasa de liberación de levofloxacino fue alta, por lo tanto, este mecanismo de liberación del fármaco sintetizado ofrece un potencial interesante como vía alterna de administración, de manera directa al órgano de infección. Este estudio de liberación in vitro arrojó resultados esperanzadores debido a que el alginato prolonga el tiempo de contacto con el epitelio diana y mejora su absorción, demostrando ser un medio eficiente de administración del fármaco (Balaji et al., 2015).

Por otro lado, una de las áreas donde se ha centrado la investigación durante los últimos años es el de la regeneración ósea. Si bien hoy en día existen variados tratamientos para las fracturas aún queda mucho por estudiar sobre 
URREJOLA, M. C.; SOTO, L. V.; ZUMARÁN, C. C.; PEÑALOZA, J. P.; ÁLVAREZ, B.; FUENTEVILLA, I. \& HAIDAR, Z. S. Sistemas de nanopartículas poliméricas I: de biodetección y monitoreo de glucosa en diabetes a bioimagen, nano-oncología, terapia génica, ingeniería de tejidos / regeneración a nano-odontología. Int. J. Morphol., 36(4):1490-1499, 2018.

los andamios y la regeneración ósea. El hueso es uno de los órganos con capacidades más espontáneas de regeneración y su tejido conectivo es sumamente dinámico. Es necesario mantener el equilibrio de la matriz ósea, lo que requiere la acción coordinada de distintos tipos celulares. Los osteblastos son las células encargadas de producir hueso, mientras los osteocitos y osteoclastos permiten la reabsorción del hueso, y con ello la remodelación ósea.

Uno de los principales problemas de los tratamientos convencionales, cuando se trata de injertos autólogos, corresponden a la morbilidad del donante y el aumento de los costos. Esto crea la necesidad de buscar nuevos tratamientos y es en esta búsqueda que se ha logrado desarrollar nuevos métodos de ingeniería ósea, como (1) terapia génica: asociada a transducción de citoquinas en los sitios de reparación; (2) Terapia de células madre: transplante de células osteogénicas cultivadas derivadas del hueso huésped; y (3) Terapia de Proteínas: aplicación de terapia osteoinductiva asociada a factores de crecimiento (Olate \& Haidar, 2017). Sin embargo, la principal protagonista de las actuales terapias para la regeneración ósea, es la terapia con células madres y genética con proteínas, la cual incorpora directamente morfogenes osteogénicos, como las proteínas morfogenéticas óseas (BMPs), las cuales son potentes citoquinas capaces de inducir nueva formación ósea in vivo e in vitro. Estas han sido eficaces en la reconstrucción de huesos largos, columna vertebral y esqueleto craneofacial. Algunos estudios en esta área han intentado manipular la regeneración ósea mediante BMP-2 (InductOs ${ }^{\circledR}$, Infuse ${ }^{\circledR}$ ) y BMP-7/OP-1 (Osifraft@) (Olate \& Haidar).

Anualmente, según el último informe de la Organización Mundial de la Salud, se estima que mueren 265.000 personas a causa de quemaduras, la mayor incidencia de estas es principalmente en países de ingresos bajos y medios, donde los supervivientes enfrentan una vida de morbilidad, gran parte de estas muertes son el resultado de las heridas producidas por las quemaduras y las infecciones asociadas, sobre todo cuando se ven comprometidas áreas extensas. Al ser destruida la barrera cutánea, aumenta la disponibilidad de nutrientes para las bacterias, lo que, sumado a la destrucción del suministro de la vasculatura y la inmunosupresión sistémica, hacen que el área sea muy susceptible a infecciones. Por lo tanto, las quemaduras plantean un problema muy complejo en la atención médica, ya que se presentan una alta susceptibilidad a la infección microbiana junto con la necesidad de lograr una cicatrización rápida y satisfactoria de las heridas y, al mismo tiempo, evitar las cicatrices indeseables. Debido a esto, generalmente, se emplean antimicrobianos tópicos para inhibir las infecciones que se producen post-quemaduras, seguidas de una terapia de antibióticos sistémicos.
La nanotecnología ha proporcionado una gama de nanoestructuras, que se pueden utilizar en aplicaciones terapéuticas y de diagnóstico en quemaduras. Las terapias incluyen NPs poliméricas, hidrogeles, NPs cerámicas, NPs metálicos, $\mathrm{NPs} \mathrm{TiO}_{2}$, NPs basados en carbono, QDs y nanofibras, utilizados como posibles agentes antimicrobianos en las quemaduras (Mofazzal Jahromi et al.). Mofazzal Jahromi et al. se centran en realizar una revisión exhaustiva de las NPs orgánicas y no orgánicas diseñadas, que tiene como finalidad proporcionar factores de crecimiento en pieles quemadas, mediante el uso, de diversas plataformas basadas en nanopartículas, las cuales pueden estar dirigidas a mejorar la cicatrización de heridas por quemaduras tales como andamios en 3D, apósitos y películas para heridas, estrategias para proporcionar factores de crecimiento y nanopartículas sensibles a los estímulos. Estas diversas plataformas pueden utilizarse eficazmente para distintas terapias para heridas de quemaduras, cómo administrar fármacos, terapia celular, administración de factores de crecimiento y terapia génica (Mofazzal Jahromi et al.). Actualmente se está explorando una amplia gama de nanoestructuras sofisticadas en heridas por quemaduras, que pueden aportar a disminuir los problemas ocasionados por las infecciones, al tiempo que estimulan la regeneración de la piel.

Nano-odontología. Actualmente la nano-odontología se ha desarrollado en base a la adquisición de nuevos materiales, y en mejorar aquellos que ya se encuentran disponibles. Algunos nano-materiales sintetizados en base a polímeros, moléculas metálicas e inorgánicas, proveen una mejora en la calidad de los cuidados en la higiene, propiedades mecánicas, antibacterianas, fluorescencia, antitumorales, remineralizantes y regenerativas.

La caries dental es causada principalmente por un microorganismo llamado Streptococcus mutans, por lo tanto, el control efectivo de éste patógeno es crítico en la prevención y tratamiento de esta enfermedad (Wang et al., 2017). El nano compuesto de $\mathrm{Ag} / \mathrm{ZnO}$ es un eficiente agente antibacteriano de baja toxicidad, este fue utilizado en un estudio para evaluar la efectividad que tiene un nano-compuesto con nanopartículas de $\mathrm{Ag} / \mathrm{ZnO}$ frente a $S$. mutans, comparándolo con un nano-compuesto de $\mathrm{Zn}$. Los resultados mostraron que el nano-compuesto con nanopartículas de $\mathrm{Ag} / \mathrm{ZnO}$ poseía una mayor eficacia antibacteriana sobre $S$. mutans, cuyo mecanismo de acción incluye la destrucción de la membrana celular y generación de especies reactivas de oxígeno que oxidan las macromoléculas (Wang et al.) (Fig. 2).

En otro trabajo, investigadores quisieron dotar al titanio con propiedades antibacterianas, para ello utilizaron nanotubos de dióxido de titanio anodizado $\left(\mathrm{TiO}_{2}\right)$, cuyas 

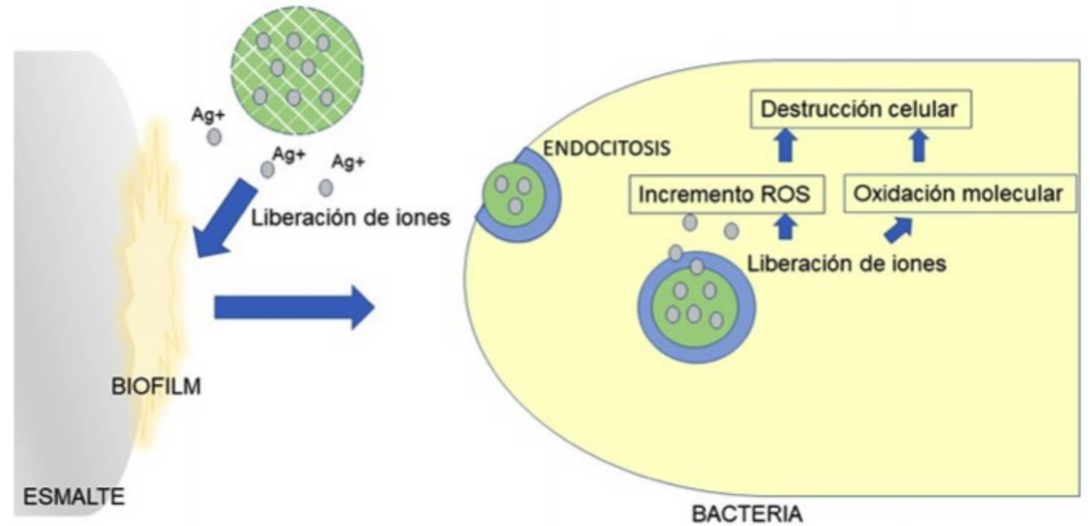

Fig. 2. Mecanismo de acción antibacteriana de Nps-Ag . Muestra los efectos de las nanopartículas de plata sobre bacterias.

paredes fueron impregnadas con nanopartículas de $\mathrm{ZnO}$ con tamaños que oscilaban entre los 20 a $50 \mathrm{~nm}$, las cuales se adhirieron estrechamente a las paredes de los nanotubos. Los resultados mostraron que el $\mathrm{Zn}$ fue liberado de los nanotubos de manera constante y lenta, manteniéndose estable los primeros 7 días. Aquellos nanotubos que conta- ban con $\mathrm{ZnO}$ inhibieron el crecimiento de $S$. mutans y $P$. gingivalis, proporcionando así propiedades antibacterianas para prevenir la infección de implantes. En el caso de $S$. mutans demostraron que inhibían la expresión de genes de adhesión bacteriana (Liu et al., 2014).

Vargas-Reus et al. (2012) evaluaron la actividad antimicrobiana de seis nanopartículas de metal y óxido metálico, y analizaron dos de sus compuestos contra los patógenos asociados en la peri-implantitis; un proceso inflamatorio que causa la pérdida del hueso de soporte y que conduce a la pérdida del implante dental. Evaluaron la actividad de diferentes nanopartículas, los que resultaron tener mejores propiedades antimicrobianas fueron aquellas con plata $(\mathrm{Ag})$, óxido cuproso $\left(\mathrm{Cu}^{2} \mathrm{O}\right)$, óxido cúprico $(\mathrm{CuO})$ y óxido de cinc $(\mathrm{ZnO})$. Por lo tanto, el recubrimiento de superficies de titanio de implantes dentales con nanopartículas

Tabla I. Nuevos nano-sistemas para medir la glucosa. Resume los nuevos nanosistemas reportados en la literatura para medir los niveles de glucosa.

\begin{tabular}{|c|c|c|c|}
\hline Tipo & Detección principal & $\begin{array}{l}\text { Tiempo de } \\
\text { respuesta }\end{array}$ & Límite de detección \\
\hline \multirow[t]{8}{*}{ Óptica } & Emisión de nanotubos cerca-IR & $\sim 1 \mathrm{~min}$ & $34,7 \mathrm{uM}$ \\
\hline & Mejora de fluorescencia de nanotubos & $\sim 1 \mathrm{~min}$ & $2.5 \mathrm{mM}$ \\
\hline & $\begin{array}{l}\text { Aumento de la fluorescencia y Crecimiento de } \\
\text { NP de Au }\end{array}$ & $30-60 \mathrm{~min}$ & $0,01 \mathrm{nM}(\mathrm{QDs}) ; 0,1 \mathrm{mM}(\mathrm{Au} \mathrm{NPs})$ \\
\hline & Actividad catalítica del grafeno & $1 \mathrm{~h}$ & $1 \mathrm{uM}$ \\
\hline & Mejora de fluorescencia de nanotubos & $\sim 1 \mathrm{~min}$ & $5 \mathrm{mM}$ \\
\hline & Espectroscopia de Roman & $\sim 10 \mathrm{~min}$ & $0,5 \mathrm{uM}$ \\
\hline & Difracción de Bragg mediada por hidrogel & $5 \mathrm{~min}$ & $90 \mathrm{uM}$ \\
\hline & Señal de proteína FRET & $\sim 1 \mathrm{~min}$ & $25 \mathrm{uM}$ \\
\hline \multirow[t]{9}{*}{ Eléctrica } & Modulación de conducta ncia de nanotubos & $\sim 20$ seg & $0,1 \mathrm{mM}$ \\
\hline & $\begin{array}{l}\text { Catálisis de peróxido de hidrógeno a través de } \\
\text { nanotubos }\end{array}$ & $<20 \mathrm{seg}$ & $0,08 \mathrm{mM}$ \\
\hline & Catálisis de peróxido de hidrogeno & $<5 \mathrm{seg}$ & $1,5 \mathrm{uM}$ \\
\hline & $\begin{array}{l}\text { Catálisis de peróxido de hidrógeno a través de } \\
\text { NPs de } \mathrm{Au}\end{array}$ & $<30$ seg & $180 \mathrm{uM}$ \\
\hline & $\begin{array}{l}\text { Nanomaterial mejorado } \quad \text { conducción } \\
\text { modulación }\end{array}$ & $<20 \mathrm{seg}$ & $0,5 \mathrm{uM} ; 5 \mathrm{uM}$ \\
\hline & $\begin{array}{l}\text { Nanomaterial mejorado } \quad \text { conducción } \\
\text { modulación }\end{array}$ & $<20$ seg & $0,56 \mathrm{mM} ; 0,26 \mathrm{mM}$ \\
\hline & Catálisis de glucosa libre de e nzimas & $<20 \mathrm{seg}$ & $<25 \mathrm{~nm}$ \\
\hline & $\begin{array}{l}\text { Catálisis de nanopartículas de peróxido de } \\
\text { hidrogeno }\end{array}$ & 3 seg & $0,7 \mathrm{uM}$ \\
\hline & Catálisis de glucosa libre de e nzimas & $<20 \mathrm{seg}$ & $1 \mathrm{mM}$ \\
\hline Magnética & Resonancia magnética de desplazamiento de & $<1 \min$ & - \\
\hline
\end{tabular}


URREJOLA, M. C.; SOTO, L. V.; ZUMARÁN, C. C.; PEÑALOZA, J. P.; ÁLVAREZ, B.; FUENTEVILLA, I. \& HAIDAR, Z. S. Sistemas de nanopartículas poliméricas I: de biodetección y monitoreo de glucosa en diabetes a bioimagen, nano-oncología, terapia génica, ingeniería de tejidos / regeneración a nano-odontología. Int. J. Morphol., 36(4):1490-1499, 2018.

antimicrobianas debe conducir a una mayor tasa de éxito de los implantes (Vargas-Reus et al.). Un estudio comparativo realizado por Ahrari et al., (2015) evaluó los efectos antibacterianos de diferentes soluciones coloidales que contienen óxido de cinc $(\mathrm{ZnO})$, óxido de cobre $(\mathrm{CuO})$, dióxido de titanio $\left(\mathrm{TiO}_{2}\right.$ ) y plata $(\mathrm{Ag})$. Compararon los efectos antimicrobianos sobre $S$. mutans y $\mathrm{S}$. sanguis, con clorhidrato de clorhexidina y flúor sódico. Los resultados mostraron que la solución que contenía nanopartículas de $\mathrm{TiO}_{2}$ fue la que mostró mayor acción antimicrobiana en $S$. mutans y $S$. sanguis, por lo tanto, puede ser investigado como alternativa al uso de clorhexidina (Ahrari et al.,).

Por otra parte, un estudio in vitro formuló adhesivos con diferentes concentraciones de nanopartículas de cobre, el cual adicionó actividades antimicrobianas en todas las concentraciones. Además, no se observó reducción de la dentina bajo la restauración ni filtración incluso después de 1 año, además de preservar la unión a la dentina sin reducir sus propiedades mecánicas (Gutiérrez et al., 2017).

Finalmente, una manera eficaz para aliviar la sensibilidad dentaria es mediante la oclusión de los túbulos dentinarios. Lin et al. (2017) fabricaron dendrímeros de poliamidoamina funcionalizados con nano-hidroxiapatita (n-HAP) y sus resultados sugieren una unión con las fibras de colágeno logrando una oclusión efectiva de los túbulos dentinarios, lo cual sugiere ser un material biocompatible y terapéutico para detener la sensibilidad dentinaria (Lin et al.). Por lo tanto, la composición básica de esta nueva nanotecnología consiste en aplicar a materiales ya conocidos, partículas inorgánicas que proveen a la estructura dentaria una mejora en la resistencia mecánica, estética, remineralización y liberación de fármacos bioactivos, que poseen propiedades anti-inflamatorias, antibióticas o antimicrobianas para su uso en materiales dentales y en el cuidado de la higiene oral, fundamental para la prevención de la caries dental.

\section{CONCLUSIÓN}

Los aspectos presentados en las presentes revisiones son sólo una pequeña fracción de las investigaciones que son realizadas hoy en día. Actualmente el campo terapéutico de nanotecnología es muy amplio, así también como sus aplicaciones. Las nano-tecnologías basadas en nanopartículas ofrecen un armazón eficiente y variable que pueden ser utilizadas para detectar patologías y también mejorar tratamientos, fortaleciendo la eficacia de los fármacos, incluso de los ya usados de manera convencio- nal. En muchas de ellas se puede mejorar la especificidad de la terapia, controlar la liberación y aumentar la vida media de los fármacos, por lo tanto, se convierten en una poderosa herramienta capaz de reducir efectos adversos indeseables y mejorar la calidad de vida de muchas personas.

\section{AGRADECIMIENTOS}

Este trabajo fue apoyado por las subvenciones de funcionamiento otorgadas al BioMAT'X (Laboratorio de Biomateriales, Farmacéuticos y Bioingeniería de Tejidos Cráneo Máxilo-Facial), miembro del CIIB (Centro de Investigación e Innovación Biomédica), a través de la Facultad de Odontología y Fondo de Ayuda a la Investigación (FAI No. INV-IN-2015-101 2015-2019), Dirección de Investigación, Universidad de los Andes, Santiago de Chile y CONICYT-FONDEF (Grant ID \# 16I10366).

URREJOLA, M. C.; SOTO, L. V.; ZUMARÁN, C. C.; PEÑALOZA, J. P.; ÁlVAREZ, B.; FUENTEVILLA, I. \& HAIDAR, Z. S. Polymer Nanoparticle Systems: from Biodetection and Glucose Monitoring in Diabetes to Bioimaging, Nano-Oncology, Gene Therapy, Tissue Engineering / Regeneration to Nano-Dentistry. Int. J. Morphol., 36(4):14901499, 2018.

SUMMARY: Nanotechnology is the science that involves the synthesis of materials in scale between 1-100 nm (nanomaterials) and is applicable in different areas such as environment, electronics, food, energy, among others. The fields that will be relevant within this review and explained in detail are nanomedicine and nano-dentistry. Currently, in these areas, the three main topics under development are specifically in the sub-area of nanobiotechnology and correspond to: sensorization (biosensors / biosensing), diagnostics (biomarkers / bioimaging) and transport of genes, proteins or drugs (exchange systems) controlled in systemic versus localized targets). Advances have also been presented in bioapplications such as membrane modeling, cell marking, delivery of agents to specific targets, strategies for disease prevention, tissue engineering, organ regeneration, immunoassay strategies and nano-oncology. This review article aims to address some of the most relevant contributions, some of the recent work, on nanoparticle systems, mainly those aimed at therapies in areas such as diabetes, nanooncology, drug and gene therapy, through the layer-by-layer and self-assembled technique, also widely used in tissue engineering and tissue regeneration, together with a brief summary of the advances that exist in the field of nano-dentistry.

KEY WORDS: Glucose; Quantum dots; Polymers; Regeneration; Nanotechnology 
URREJOLA, M. C.; SOTO, L. V.; ZUMARÁN, C. C.; PEÑALOZA, J. P.; ÁLVAREZ, B.; FUENTEVILLA, I. \& HAIDAR, Z. S. Sistemas de nanopartículas poliméricas I: de biodetección y monitoreo de glucosa en diabetes a bioimagen, nano-oncología, terapia génica, ingeniería de tejidos / regeneración a nano-odontología. Int. J. Morphol., 36(4):1490-1499, 2018.

\section{REFERENCIAS BIBLIOGRÁFICAS}

Ahrari, F.; Eslami, N.; Rajabi, O.; Ghazvini, K. \& Barati, S. The antimicrobial sensitivity of Streptococcus mutans and Streptococcus sangius to colloidal solutions of different nanoparticles applied as mouthwashes. Dent. Res. J.(Isfahan), 12(1):44-9, 2015.

Ancla, C.; Lapeyre, V.; Gosse, I.; Catargi, B. \& Ravaine, V. Designed glucose-responsive microgels with selective shrinking behavior. Langmuir, 27(20):12693-701, 2011.

Balaji, R. A.; Raghunathan, S. \& Revalthy, R. Levofloxacin: formulation and in-vitro evaluation of alginate and chitosan nanospheres. Egypt. Pharm.J., 14(1):30-5, 2015.

Chatterjee, K.; Sarkar, S.; Rao, K. J. \& Paria, S. Core/shell nanoparticles in biomedical applications. Adv. Colloid Interface Sci., 209:8-39, 2014.

DiSanto, R. M.; Subramanian, V. \& Gu, Z. Recent advances in nanotechnology for diabetes treatment. Wiley Interdiscip. Rev. Nanomed. Nanobiotechnol., 7(4):548-64, 2015.

Field, L.; Walper, S. A.; Susumu, K.; Oh, E.; Medintz, I. L. \& Delehanty, J. B. Semiconductor quantum-dots as Förster resonance energy transfer donors for intracellularly-based biosensors. In: Osinski, M.; Parak, W. J. \& Liang, X. J.Colloidal Nanoparticles for Biomedical Applications XII. Proc. SPIE, 10078:100780N-1, 2017.

Goldberg, M. S. Immunoengineering: how nanotechnology can enhance cancer immunotherapy. Cell, 161(2):201-4, 2015.

Gutiérrez, M.F.; Malaquias, P.; Matos, T. P.; Szesz,A.; Souza, S.; Bermudez, J.; Reis, A.; Loguercio, A. D. \& Farago, P. V. Mechanical and microbiological properties and drug release modeling of an etch-andrinse adhesive containing copper nanoparticles. Dent. Mater., 33(3):30920, 2017.

Irvine, D. J.; Swartz, M. A. \& Szeto, G. L. Engineering synthetic vaccines using cues from natural immunity. Nat. Mater., 12(11):978-90, 2013.

Jeevanandam, J.; Chan, Y. S. \& Danquah, M. K. Nano-formulations of drugs: Recent developments, impact and challenges. Biochimie, 1289:99-112, 2016.

Jie, G.; Zhao, Y. \& Qin, Y. A fluorescent polymeric quantum dot/aptamer superstructure and its application for imaging of cancer cells. Chem. Asian J., 9(5):1261-4, 2014.

Li, S.; Xu, L.; Sun, M.; Wu, X.; Liu, L.; Kuang, H. \& Xu, C. Hybrid nanoparticle pyramids for intracellular dual MicroRNAs biosensing and bioimaging. Adv. Mater., 29(19), 2017.

Lin, X.; Xie, F.; Ma, X.; Hao, Y.; Qin, H. \& Long, J. Fabrication and characterization of dendrimer-functionalized nano-hydroxyapatite and its application in dentin tubule occlusion. J. Biomater. Sci.Polym.Ed., 28(9):846-63, 2017.

Liu, H.; Tang, W.; Li, C.; Lv, P.; Wang, Z.; Liu, Y.; Zhang, C.; Bao, Y.; Chen, H.; Meng, X.; Song, Y.; Xia, X.; Pan, F.; Cui, D. \& Shi, Y. CdSe/ $\mathrm{ZnS}$ quantum dots-labeled mesenchymal stem cells for targeted fluorescence imaging of pancreas tissues and therapy of type 1 diabetic rats. Nanoscale Res. Lett., 10(1):959, 2015.

Liu, W.; Su, P.; Chen, S.; Wang, N.; Ma, Y.; Liu, Y.; Wang, J.; Zhang, Z.; Li, H. \& Webster, T. J. Synthesis of TiO2 nanotubes with $\mathrm{ZnO}$ nanoparticles to achieve antibacterial properties and stem cell compatibility. Nanoscale, 6(15):9050-62, 2014.

Mofazzal Jahromi, M. A.; Sahandi Zangabad, P.; Moosavi Basri, S. M.; Sahandi Zangabad, K.; Ghamarypour, A.; Aref, A. R.; Karimi, M. \& Hamblin, M. R. Nanomedicine and advanced technologies for burns: Preventing infection and facilitating wound healing. Adv. Drug Deliv. Rev., 123:33-64, 2018.

Mukhopadhyay, P.; Chakraborty, S.; Bhattacharya, S.; Mishra, R. \& Kundu, P.P. pH-sensitive chitosan/alginate core-shell nanoparticles for efficient and safe oral insulin delivery. Int. J. Biol. Macromol., 72:640-8, 2015.

Olate, S. M. \& Haidar, Z. S. Growth factor-assisted distraction osteogenesis and histiogenesis. J. Oral Res., 6(5):112-4, 2017.

Quent, V.; Taubenberger, A.; Reichert, J. C.; Martine, L. C.; Clements, J.
A.; Hutmacher, D. W. \& Loessner, D. A humanised tissue-engineered bone model allows species-specific breast cancer-related bone metastasis in vivo. J. Tissue Eng. Regen. Med., 12(2):494-504, 2017.

Stephan, S. B.; Taber, A. M.; Jileaeva, I.; Pegues, E. P.; Sentman, C. L. \& Stephan, M. T. Biopolymer implants enhance the efficacy of adoptive T-cell therapy. Nat. Biotechnol., 33(1):97-101, 2015.

Topalian, S. L.; Weiner, G. J. \& Pardoll, D. M. Cancer immunotherapy comes of age. J. Clin. Oncol., 29(36):4828-36, 2011.

Vargas-Reus, M. A.; Memarzadeh, K.; Huang, J.; Ren, G. G. \& Allaker, R. $\mathrm{P}$. Antimicrobial activity of nanoparticulate metal oxides against periimplantitis pathogens. Int. J. Antimicrob. Agents, 40(2):135-9, 2012.

Veiseh, O.; Tang, B. C.; Whitehead, K. A.; Anderson, D. G. \& Langer, R. Managing diabetes with nanomedicine: challenges and opportunities. Nat. Rev. Drug Discov., 14(1): 45-57, 2015.

Wang, S.; Wu, J.; Yang, H.; Liu, X.; Huang, Q. \& Lu, Z. Antibacterial activity and mechanism of $\mathrm{Ag} / \mathrm{ZnO}$ nanocomposite against anaerobic oral pathogen Streptococcus mutans. J. Mater. Sci. Mater. Med., 28(1):23, 2017.

Ye, D.; Liang, G.; Li, H.; Luo, J.; Zhang, S.; Chen, H. \& Kong, J. A novel nonenzymatic sensor based on $\mathrm{CuO}$ nanoneedle/graphene/carbon nanofiber modified electrode for probing glucose in saliva. Talanta, 116:223-30, 2013.

\section{Dirección para correspondencia: \\ Ziyad S. Haidar \\ Professor and Scientific Director \\ Faculty of Dentistry \\ Universidad de Los Andes \\ Las Condes \\ Santiago \\ CHILE}

Recibido : 15-05-2018

Aceptado: 30-09-2018

E-mail: zhaidar@uandes.cl 